\title{
Editorial: New Horizons in Genetic Diagnosis in Pediatric Practice: The Excitement and Challenges!
}

\author{
Ratna D. Puri ${ }^{1}$ • Madhulika Kabra ${ }^{2}$
}

Received: 7 July 2016 / Accepted: 18 July 2016/Published online: 11 August 2016

(C) Dr. K C Chaudhuri Foundation 2016

Establishing a genetic diagnosis has always been interesting and exciting. It could just be a spot diagnosis of a rare genetic syndrome, suspecting a neurodegenerative disorder on MRI findings or interpreting a complex karyotype. Earlier with limited availability and access to genetic diagnostic testing, though life was simpler, a significant proportion of patients remained "idiopathic" or undiagnosed.

In the post genomic era medical genetics has come a long way and the advances in technology have brought about a tremendous change in its office practice. Genetic diagnosis no longer is confined only to clinical examination, karyotyping, neuroimaging, screening for metabolic disorders and few targeted molecular tests. Investigations have become more sophisticated and exploratory, as many high-end technologies are easily available almost over the counter. This statement does not in any way undermine the importance of a detailed phenotypic evaluation and adjunct baseline testing to establish a set of differential diagnosis even in this new era. No high-end technology and tests can ever replace the brainpower and experience of a clinician. Investigations add on to confirm a suspected diagnosis. Moreover these newer tests, though now available, are very expensive and difficult to interpret at times, emphasizing the importance of a detailed clinical evaluation and narrowing down the clinical possibilities before ordering any of these newer investigations propagated as "umbrella tests". The excitement and curiosity (that is

Madhulika Kabra

madhulikakabra@hotmail.com

1 Institute of Medical Genetics and Genomics, Sir Ganga Ram Hospital, New Delhi, India

2 Division of Genetics, Department of Pediatrics, All India Institute of Medical Sciences, New Delhi 110029, India natural) to establish a conclusive diagnosis (that is not always possible) often overpowers the known and unknown challenges ahead that are only conceived when the test results are in hand. Thus it is imperative for the physician to understand the test being ordered, its limitations, the implications and the importance of pre and post test counseling.

The diagnostic armamentarium has expanded from karyotyping to chromosomal microarray and from single gene testing to Next Generation Sequencing (NGS) used for a panel of genes, clinical exome, whole exome and whole genome sequencing. While it looks fascinating and promises to answer all patient related queries, practically we now appreciate that we are far from this. The vast array of available genetic tests poses a challenge not only for a pediatrician, but at times even for a clinical geneticist. It may be difficult at times to decide that which is the most appropriate test in a particular clinical setting. Also interpretation of some of the ambiguities of the analysis, and finally, communication of these uncertainties to the families is quite challenging [1]. To keep pace with and utilize these fast emerging genetic technologies appropriately, a minimum basic genetic knowledge is required for all clinicians, especially pediatricians, in whose ambit many genetic diagnostic challenges lie. They particularly are the ones likely to order and interpret genetics test results [2]. This is the current scenario in India as well, with all the advanced genetic tests being propagated by companies in the absence of adequately trained genetic counselors and clinicians to guide patients and families about the appropriate test, its utility and limitations.

These newer technologies are useful in some common situations of uncertain genetic etiology like autism and idiopathic intellectual disability, and also for disorders with a definite mendelian inheritance but extensive genetic heterogeneity (e.g., deafness and retinitis pigmentosa), very large genes (e.g., cystic fibrosis, hemophilia, neurofibromatosis) or 
disorders with possible genetic etiology with or without a family history [3]. Newer genetic techniques often improve the diagnostic yield in such situations, if used judiciously and interpreted correctly.

As more and more clinicians are getting exposed (but not necessarily educated) to these technologies, this issue of Indian Journal of Pediatrics brings out a balanced approach for genetic diagnosis for selected common disorders seen in clinical practice. The current symposium on "New horizons in genetic diagnosis in pediatric practice" was conceived with the aim to provide an overview of the exciting advances and their clinical applications. Discussed within the purview of a clinical approach for commonly encountered situations that may not have a clear diagnostic end point, this symposium promises to be useful and exciting for pediatricians. All the articles have emphasized the need for a thorough clinical evaluation and targeted testing before proceeding to the more complex testing in a step wise manner. The disorders chosen for this symposium, though largely unrelated, but relatively common in day to day practice, emphasize that following a structured clinical and investigational step wise approach is far reaching than a blanket approach. Establishing a genetic diagnosis in these disorders not only puts an end to the expensive and apprehension driven "diagnostic odyssey" for the families, but also helps them to understand, come to terms, and deal with the genetic disorder in their child/family, the prognosis and treatment options, if any. The exciting times of technological advances have not only seen improvement in diagnosis but also therapeutic options for genetic disorders, and therefore not making a definite diagnosis can possibly do harm and create lost opportunities for treatment. Diagnosis is also important for providing appropriate genetic counseling, understanding the inheritance and recurrence risks and offering families suitable reproductive options and prenatal diagnosis to prevent recurrence if desired and indicated.

Autism is a very common but complex disorder, both in identifying the etiology and management. Even though a genetic etiology may be identified in a small proportion of cases, clinicians should be aware about the approach and yield of genetic tests in autism in view of its high prevalence [4].

Intellectual disability (ID), a highly prevalent problem encountered in clinical practice, again poses a challenge for the clinician due to its etiological heterogeneity. The article on ID focuses on step wise approach, emphasizes on good clinical evaluation and impact of newer techniques in deciphering the etiology [5].

Deafness, reported to be the most common sensory disability has been included to appraise the clinician about the approach for reaching to a confirmatory diagnosis emphasizing the revolutions in the field of molecular testing with cutting edge technologies which have reached from bench to bedside [6].

Mitochondrial disorders are difficult to diagnose but are suspected frequently in Pediatric practice. The article on mitochondrial disorders aims to give a simplified approach to these cases, explaining the complexities in making a confirmed diagnosis and counseling [7].

Epileptic encephalopathies commonly encountered in Pediatric Neurology practice are a challenge not only for treating but in making an etiological diagnosis. The article focuses on genetic etiologies in this group of disorders with emphasis on inherited metabolic disorders [8].

The article on overgrowth syndromes, gives a structured approach to the problem, underlining the importance of defining a clinical phenotype and steps for identifying a genetic etiology [9].

In the present era it is becoming increasingly important for clinicians to understand basics of genetic testing as with increasing awareness it is likely that they will come across more such patients. It is important to have simple guidelines to approach selected relatively common genetic disorders which will help the clinician to do initial work up before referral to appropriate facility if needed. This knowledge would guide the clinicians to avoid misuse of these expensive and complex tests and help to identify appropriate situations where these are likely to be beneficial.

\section{Compliance with Ethical Standards}

Conflict of Interest None.

Source of Funding None.

\section{References}

1. McPherson E. Genetic diagnosis and testing in clinical practice. Clin Med Res. 2006;4:123-9.

2. Katsanis HS, Katsanis N. Molecular genetic testing and the future of clinical genomics. Nat Rev Genet. 2013;14:415-26.

3. Stavropoulos DJ, Merico D, Jobling R, et al. Whole-genome sequencing expands diagnostic utility and improves clinical management in paediatric medicine. Genom Med. 2016;1:15012. doi:10.1038/npjgenmed.2015.12.

4. Sudarshan S, Gupta N, Kabra M. Genetic studies in autism. Indian J Pediatr. 2016. doi:10.1007/s12098-015-1989-7.

5. Puri RD, Tuteja M, Verma IC. Genetic approach to diagnosis of intellectual disability. Indian J Pediatr. 2016. doi:10.1007/s12098016-2205-0.

6. Carey JC, Palumbos JC. Advances in the understanding of the genetic causes of hearing loss in children inform a rational approach to evaluation. Indian J Pediatr. 2015. doi:10.1007/s12098-015-1941-x.

7. Korenev S, Morris AAM. When to suspect and how to diagnose mitochondrial disorders? Indian J Pediatr. 2016. doi:10.1007 /s12098-015-1932-y.

8. Patel J, Mercimek-Mahmutoglu S. Epileptic encephalopathy in childhood: a stepwise approach for identification of underlying genetic causes. Indian J Pediatr. 2016. doi:10.1007/s12098-015-1979-9.

9. Suri M. Approach to the diagnosis of overgrowth syndromes. Indian J Pediatr. 2015. doi:10.1007/s12098-015-1958-1. 